\title{
Serum cholesterol, haemorrhagic stroke, ischaemic stroke, and myocardial infarction: Korean national health system prospective cohort study
}

\author{
Shah Ebrahim, Joohon Sung, Yun-Mi Song, Robert Ferrer, Debbie A Lawlor, George Davey Smith
}

\begin{abstract}
Objective To investigate risk factors, such as heavy alcohol consumption, that might explain any increased risk of haemorrhagic stroke associated with low blood cholesterol. Design Prospective cohort study.

Setting Korea.

Participants 787442 civil servants (661 700 men, 125742 women) aged 30-64.

Main outcome measures Cardiovascular risk factors were assessed at biennial health check. Data on morbidity and mortality were ascertained from 1990 to 2001 using hospital admissions and mortality surveillance systems.

Results 6328 cases of ischaemic stroke (6021 men, 307 women), 3947 cases of haemorrhagic stroke (3748 men, 199 women), 3170 cases of undefined stroke (2902 men, 268 women), and 4417 cases of myocardial infarction (4305 men, 112 women) occurred. Ischaemic stroke and myocardial infarction were strongly and positively associated with blood cholesterol (hazard ratio per $1 \mathrm{mmol} / \mathrm{l}$ cholesterol $1.20(95 \%$ confidence 1.16 to 1.24 ) and 1.48 (1.43 to 1.53 ), respectively). Haemorrhagic stroke showed an inverse association in fully adjusted models (0.91, 0.87 to 0.95$)$. This inverse association was confined to participants with hypertension. When stratified by concentration of $\gamma$ glutamyl transferase (GGT), an indicator of alcohol consumption, the association was not seen in participants with low concentrations of GGT, and it was independent of hypertension in those with high concentrations of GGT (>80 U/l).

Conclusion High alcohol consumption may underlie the association between low blood cholesterol and increased risk of haemorrhagic stroke.
\end{abstract}

\section{Introduction}

Stroke is the third most common cause of death and a leading cause of severe disability in both the developed and developing world. ${ }^{1}$ The burden of stroke is becoming greater as the population ages, making its prevention a priority.

Mortality from haemorrhagic stroke declined consistently over the 20th century in many countries, but ischaemic stroke showed a rise and a fall, mirroring the coronary heart disease epidemic. ${ }^{2}$ These different secular trends indicate that the risk factors for the two subtypes of stroke may differ. Changes in major cardiovascular risk factors may explain up to two thirds of the decline in mortality from stroke. ${ }^{34}$ In Japan, even though concentrations of blood cholesterol rose from the 1960s to the 1980s, rates of ischaemic and haemorrhagic stroke have fallen dramatically. ${ }^{56}$ The importance of individual risk factors can be determined only by examining the different subtypes of stroke.

The role of blood cholesterol as a cause of stroke remains uncertain..$^{7-9}$ The lack of an association between all types of stroke and concentrations of cholesterol may mask a positive association with ischaemic stroke and an inverse association with haemorrhagic stroke, although the relation with haemorrhagic stroke may not be causal. ${ }^{10-13}$ Studies of different populations give imprecise estimates of the association between cholesterol and subtypes of stroke. ${ }^{14-16}$ The inverse association between serum cholesterol and haemorrhagic stroke may be specific to men with hypertension; this has led to speculation that heavy alcohol consumption underlies hypertension, hypocholesterolaemia, and increased risk of haemorrhagic stroke. ${ }^{17}$

This uncertainty causes concern that treatment to lower cholesterol might increase risk of haemorrhagic stroke. ${ }^{18}$ A recent review of randomised trials found no influence of such treatment on haemorrhagic stroke (relative risk 1.05, 95\% confidence interval 0.78 to 1.41 , for a $1 \mathrm{mmol} / 1$ lowering of low density lipoprotein cholesterol). ${ }^{19}$ However, only 204 cases (105 in the treatment group; 99 in the control group) were used to estimate the risk of haemorrhagic stroke, and confidence intervals were wide. We aimed to resolve this uncertainty by investigating the association of blood cholesterol with stroke subtype in a large cohort of Korean civil servants.

\section{Methods}

Participants and study measures

Participants were Korean male and female public servants aged 30-64, who had a health check provided by the Korean Medical Insurance Corporation between 1986 and 1990. In Korea, medical insurance is provided to everyone under the Korean national health system. The Korea Medical Insurance Corporation was one of the main national health insurance institutions at the time and was in charge of medical insurance services provided to all public servants and their unemployed family members. In total, 902222 people were examined (95\% of all public servants). We excluded 93540 people who changed job or had a myocardial infarction or stroke between 1986 and 1990, and 21240 people who lack data on blood cholesterol; this left a study population of 787442 (661 700 men, 125742 women). Details of the study measurements have been published..$^{20}$ Extra information on risk factors was obtained from the biennial multiphasic health examination and a self administered questionnaire. We excluded measures taken after a cardiovascular event for people who had 
Table 1 Associations between cardiovascular risk factors and cholesterol concentrations. Korean national health system study, 1986-2001. Values are mean (SD) unless stated otherwise

\begin{tabular}{|c|c|c|c|c|c|c|c|}
\hline \multirow{3}{*}{ Risk factors } & \multirow{3}{*}{$\begin{array}{l}\text { No of participants } \\
\text { available }\end{array}$} & \multicolumn{6}{|c|}{ Serum cholesterol concentration $(\mathrm{mmol} / \mathrm{l})$} \\
\hline & & & 3.36-4.13 $(n=105$ & 4.14-5.16 $(n=415$ & $5.17-6.20(n=217$ & $6.21-6.97(n=32$ & \\
\hline & & $\leq 3.36(n=8319)$ & 293) & 744) & 158) & 945) & $\geq 6.98 \quad(n=7983)$ \\
\hline Age (years) & 787442 & $40.1(9.3)$ & $39.9(8.5)$ & $41.8(8.6)$ & $43.8(8.7)$ & $45.4(8.8)$ & $46.1(8.9)$ \\
\hline Systolic blood pressure $(\mathrm{mm} \mathrm{Hg})$ & 787418 & $119.5(13.4)$ & $119.8(12.5)$ & $122.4(12.8)$ & $125.6(13.7)$ & $128.5(14.7)$ & $130.7(16.0)$ \\
\hline Diastolic blood pressure $(\mathrm{mm} \mathrm{Hg})$ & 787414 & $77.8(9.3)$ & $78.1(8.5)$ & $79.9(8.6)$ & $82.1(8.9)$ & $84.0(9.4)$ & $85.3(10.1)$ \\
\hline Body mass index & 744873 & $21.7(2.2)$ & $22.2(2.3)$ & $22.9(2.4)$ & $23.7(2.4)$ & $24.2(2.4)$ & $24.3(2.4)$ \\
\hline Glucose $\geq 7 \mathrm{mmol} / \mathrm{l}(\%)$ & 787431 & 3.3 & 2.1 & 2.8 & 5.1 & 8.9 & 14.9 \\
\hline$\gamma$ glutamyl transferase $(\mathrm{U} / \mathrm{l})^{*}$ & 500419 & $36.2(68.5)$ & $33.9(55.0)$ & $38.8(53.3)$ & $48.0(59.8)$ & $59.2(72.3)$ & 76.8 (99.7) \\
\hline$\gamma$ glutamyl transferase $>80 \mathrm{U} / /^{*}, \mathrm{n}(\%)$ & 500419 & $286(7.8)$ & $4491(6.9)$ & $23663(8.7)$ & $18072(13.1)$ & $3641(19.1)$ & $1082(26.6)$ \\
\hline$\geq 20$ cigarettes a day, $\mathrm{n}(\%)$ & 744413 & $552(8.7)$ & $9511(9.8)$ & $46566(11.8)$ & $29581(14.2)$ & $5014(16.3)$ & $1258(17.7)$ \\
\hline No alcohol, n (\%) & 722662 & $3643(62.4)$ & $52523(56.3)$ & $198096(51.4)$ & $97533(48.2)$ & $14137(47.9)$ & $3221(48.2)$ \\
\hline Alcohol <30 g/week, n (\%) & 722662 & $3868(66.3)$ & $56388(60.4)$ & $213967(55.6)$ & $105916(52.4)$ & $15234(51.6)$ & $3422(51.2)$ \\
\hline Alcohol $\geq 210$ g/week, n (\%) & 722662 & 798 (13.7) & $14101(15.1)$ & $66798(17.3)$ & 38697 (19.1) & $5757(19.5)$ & $1450(21.7)$ \\
\hline Regular exercise, $\mathrm{n}(\%)$ & 742973 & $932(14.7)$ & $15489(16.0)$ & $69324(17.6)$ & $39345(19.0)$ & $5800(18.9)$ & $1325(18.7)$ \\
\hline Lowest income group, $\mathrm{n}(\%)$ & 787442 & $4430(53.3)$ & 38469 (36.5) & $123498(29.7)$ & $57240(26.4)$ & $9052(27.5)$ & $2518(31.5)$ \\
\hline
\end{tabular}

${ }^{*}$ Excluding participants positive for hepatitis $B$ or of unknown status.

All risk factors were significantly different across concentrations of serum cholesterol $(P<0.001)$ by $\chi^{2}$ test, Mantel-Haenszel $\chi^{2}$ test, and ANOVA.

such an event during the study, and we used mean values between 1986 and 1996 for measures that were repeated.

Initially we classified participants into three groups according to the ATP III (adult treatment panel III) classification of cholesterol concentrations (low, medium, or high: $<5.16$ $\mathrm{mmol} / \mathrm{l}, 5.17-6.20 \mathrm{mmol} / \mathrm{l}$, or $\geq 6.21 \mathrm{mmol} / \mathrm{l}){ }^{21}$ We further divided the lower and the higher groups to give six groups in total (table 1).

We categorised fasting glucose concentrations as normal $(<7.0 \mathrm{mmol} / \mathrm{l})$ or high $(\geq 7.0 \mathrm{mmol} / \mathrm{l})$. Blood pressure was measured at each examination and classified as normal or prehypertensive $(<140 /<90 \mathrm{~mm} \mathrm{Hg})$ or hypertensive $(\geq 140$ or $\geq 90 \mathrm{~mm} \mathrm{Hg}){ }^{22}$ Participants who had a recorded diagnosis of hypertension on more than two occasions were also classified as hypertensive.

We classified smoking habits as never smoked, ex-smoker, 1-19 cigarettes a day, or $\geq 20$ cigarettes a day. Physical exercise
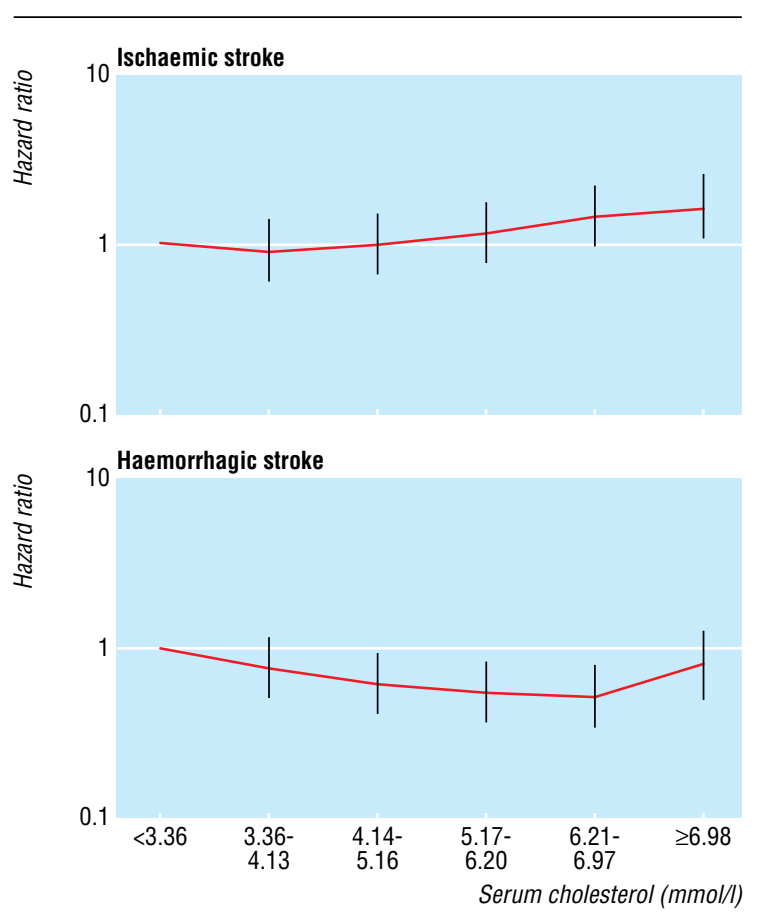

Fig 1 Fully adjusted risk of stroke in the Korean national health system study, 1986-2001 was categorised as did or did not do regular exercise. Alcohol consumption was categorised as $<30,30-104,105-209,210-419$, or $\geq 420 \mathrm{~g}$ /week. We also measured $\gamma$ glutamyl transferase (GGT) as a proxy of alcohol intake (Spearman's correlation coefficient 0.40 between self reported alcohol consumption and GGT values; $\mathrm{P}<0.0001$ ). Because participants (especially heavy drinkers) may under-report alcohol consumption, we stratified the analyses by each measure.

Hepatitis B virus antigen seropositivity was determined by reverse haemagglutination or enzyme linked immunoassay. Economic status was grouped into four categories on the basis of monthly salary in 1990.

\section{Follow-up of mortality and morbidity from stroke}

All myocardial infarctions, non-fatal strokes, and fatal strokes occurring between 1 August 1990 and 31 July 2001 were included. We used the following codes in ICD-10 (international classification of diseases, 10th revision) to identify and classify myocardial infarction and stroke: myocardial infarction (I21I24), all stroke (I60-I69), ischaemic stroke (I63, I67.8), and haemorrhagic stroke (I61). Transient ischaemic attacks and subarachnoid haemorrhages were excluded. We ascertained fatal cases from the Korean national statistical office and the death benefit record of the Korean national health system. Morbidity was ascertained from hospital admissions. Patients with non-fatal disease had probably been admitted to hospital at some stage of their illness because public servants have easy access to hospital care and we followed up the participants for 10 years.

\section{Analytical methods}

Follow-up began in August 1990, and the participants were censored at the date of admission or death attributable to stroke or myocardial infarction or other causes of death, or at 31 July 2001 if no censoring event occurred. We used Cox proportional hazards regression analysis to assess hazard ratios for myocardial infarction and subtypes of stroke according to concentrations of serum cholesterol. Results of Weibul regression analysis were compared for models where the proportionality assumption was not upheld. We used regression models for myocardial infarction, all stroke, ischaemic stroke, and haemorrhagic stroke, initially adjusting for age and sex and then for all covariates. To identify any effect of pre-existing occult disease, we repeated analyses after excluding events in the first five years of follow-up. We also stratified by presence or absence of hypertension and by 
Table 2 Risk of stroke and myocardial infarction in Korean national health system study, 1986-2001. * Values are hazard ratios (95\% confidence interval) unless stated otherwise

\begin{tabular}{|c|c|c|c|c|c|}
\hline \multirow{2}{*}{$\begin{array}{l}\text { Serum cholesterol } \\
(\mathrm{mmol} / \mathrm{l})\end{array}$} & \multirow{2}{*}{ No of participants } & \multicolumn{3}{|c|}{ Stroke } & \multirow{2}{*}{ Myocardial infarction } \\
\hline & & Allt & Ischaemic & Haemorrhagic & \\
\hline \multicolumn{6}{|c|}{ Age and sex adjusted model } \\
\hline & & $\mathrm{n}=13445$ & $\mathrm{n}=6328$ & $\mathrm{n}=3947$ & $\mathrm{n}=4417$ \\
\hline$<3.36$ & 8319 & 1.00 & 1.00 & 1.00 & 1.00 \\
\hline $3.36-$ & 105293 & 1.10 (0.89 to 1.35$)$ & $1.30(0.89$ to 1.90$)$ & 0.87 (0.63 to 1.19) & 1.06 (0.68 to 1.66$)$ \\
\hline $4.14-$ & 415744 & 1.13 (0.93 to 1.38) & 1.69 (1.17 to 2.44) & 0.81 (0.59 to 1.09) & $1.42(0.92$ to 2.19$)$ \\
\hline $5.17-$ & 217158 & 1.35 (1.10 to 1.65$)$ & 2.33 (1.61 to 3.35$)$ & 0.86 (0.63 to 1.17) & 2.44 (1.59 to 3.75$)$ \\
\hline $6.21-$ & 32945 & 1.79 (1.45 to 2.20$)$ & 3.39 (2.33 to 4.92$)$ & 0.98 (0.71 to 1.37$)$ & 3.89 (2.51 to 6.03$)$ \\
\hline$\geq 6.98$ & 7983 & 2.55 (2.04 to 3.20$)$ & 4.54 (3.07 to 6.70$)$ & 1.56 (1.08 to 2.24$)$ & 6.25 (3.98 to 9.82$)$ \\
\hline Per $1 \mathrm{mmol} / \mathrm{l}$ increa & & 1.22 (1.19 to 1.24$)$ & 1.41 (1.37 to 1.45$)$ & 1.04 (1.00 to 1.09$)$ & 1.66 (1.61 to 1.71) \\
\hline \multicolumn{6}{|c|}{ Fully adjusted model§ } \\
\hline & & $\mathrm{n}=11704$ & $\mathrm{n}=5676$ & $\mathrm{n}=3345$ & $\mathrm{n}=4004$ \\
\hline$<3.36$ & 5477 & 1.00 & 1.00 & 1.00 & 1.00 \\
\hline 3.36- & 91367 & 0.83 (0.65 to 1.07$)$ & 0.90 (0.59 to 1.39) & 0.76 (0.51 to 1.14) & 0.89 (0.51 to 1.56) \\
\hline $4.14-$ & 379907 & 0.75 (0.59 to 0.96$)$ & 0.98 (0.64 to 1.49) & $0.61 \quad(0.41$ to 0.91$)$ & $1.06(0.62$ to 1.84$)$ \\
\hline $5.17-$ & 199523 & 0.77 (0.60 to 0.98$)$ & 1.14 (0.75 to 1.73$)$ & 0.55 (0.37 to 0.83$)$ & 1.62 (0.94 to 2.80$)$ \\
\hline $6.21-$ & 28949 & 0.89 (0.69 to 1.15) & 1.46 (0.96 to 2.24) & 0.52 (0.34 to 0.79$)$ & $2.30(1.32$ to 4.00$)$ \\
\hline$\geq 6.98$ & 6484 & 1.13 (0.87 to 1.48$)$ & 1.67 (1.07 to 2.61) & 0.80 (0.50 to 1.26$)$ & 3.40 (1.93 to 5.99) \\
\hline Per $1 \mathrm{mmol} / \mathrm{l}$ increa & & 1.05 (1.03 to 1.08$)$ & 1.20 (1.16 to 1.24) & 0.91 (0.87 to 0.95$)$ & 1.48 (1.43 to 1.53$)$ \\
\hline
\end{tabular}

*All strokes ICD I60-I69; ischaemic strokes ICD I63, 167.8; haemorrhagic strokes ICD I61; myocardial infarction ICD I21-I24.

†Includes unidentified strokes: 3170 cases (2902 men, 268 women) in the age and sex adjusted model and 2683 (2426 men, 257 women) in the fully adjusted model.

$\ddagger$ Participants with data on all covariates used in fully adjusted models.

$\S$ Adjusted for age, sex, body mass index, height, serum glucose, hypertension, ethanol consumption, smoking, physical activity, monthly pay, and area of residence.

both blood pressure and blood GGT concentrations (as a proxy for alcohol consumption) to evaluate the association between cholesterol and haemorrhagic stroke. We excluded 213921 participants who had no GGT value or were positive or not tested
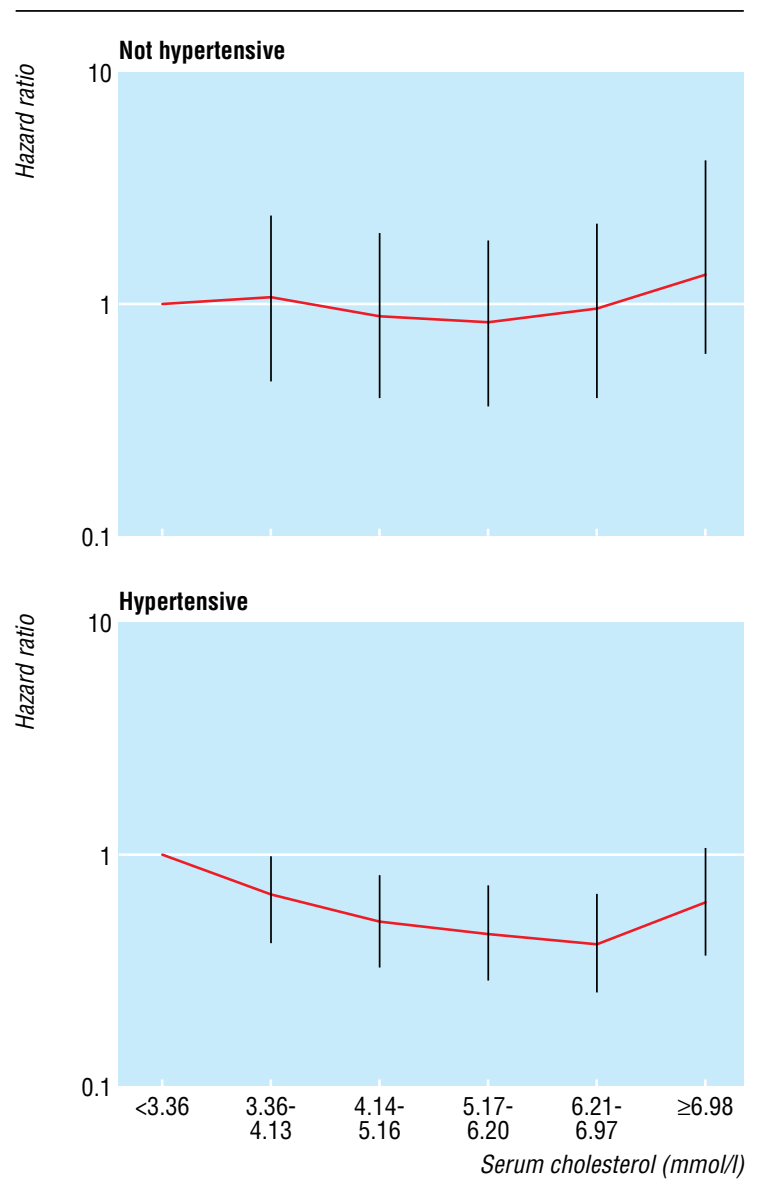

Fig 2 Fully adjusted risk of haemorrhagic stroke in the Korean national health system study, 1986-2001 for hepatitis B. The distribution of age adjusted risk factors and cholesterol associations were similar for participants who were excluded and those who were included. We could not perform sex specific analyses because female public servants were fewer and younger than their male counterparts.

\section{Results}

Of the 787442 participants (661 700 men and 125742 women), 6328 (6021 men and 307 women) had an ischaemic stroke, 3947 (3748 men and 199 women) a haemorrhagic stroke, 3170 (2902 men, 268 women) an undefined stroke, and 4417 (4305 men and 112 women) a myocardial infarction. Table 1 shows the distribution of risk factors according to serum cholesterol group. Participants with the highest concentrations of cholesterol were older and had higher blood pressure, greater body mass index, and higher fasting serum glucose values than those with lower cholesterol concentrations. Reporting of heavy drinking $(\geq 210$ $\mathrm{g}$ alcohol/week) was more prevalent among participants with higher concentrations of serum cholesterol. GGT concentration showed a J shaped association with blood cholesterol.

Table 2 shows the associations between serum cholesterol and stroke subtypes and myocardial infarction. All strokes showed a trend of increasing risk with higher cholesterol concentration (hazard ratio for highest versus lowest groups, age and sex adjusted $2.55,95 \%$ confidence interval 2.04 to 3.20 ), but this was greatly attenuated when adjusted for other covariates (1.13, 0.87 to 1.48). Examining the associations with ischaemic stroke showed a strong, linear association with serum cholesterol (age and sex adjusted 4.54, 3.07 to 6.70). Full adjustment for covariates attenuated the association with ischaemic stroke but did not completely remove this association $(1.67,1.07$ to 2.61$)$. The association with haemorrhagic stroke was non-linear, with weak evidence of an increased risk in both the lowest and the highest serum cholesterol groups. The fully adjusted hazard ratios of ischaemic stroke and myocardial infarction for each $1 \mathrm{mmol} / \mathrm{l}$ increase in serum cholesterol were 1.20 (1.16 to 1.24) and 1.48 (1.43 to 1.53). Full adjustment for covariates completely 

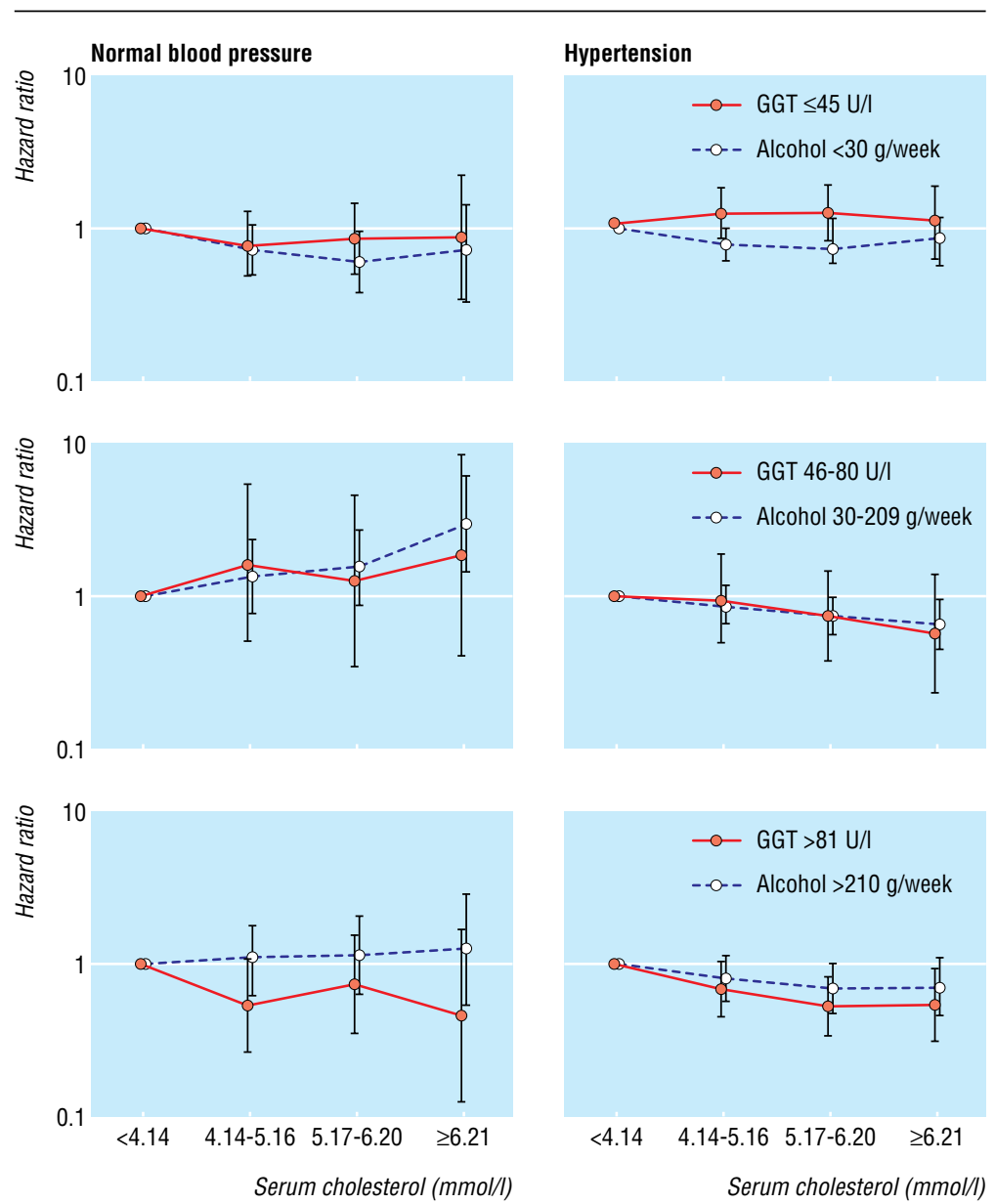

Fig 3 Fully adjusted risk for haemorrhagic stroke after participants who were seropositive for hepatitis B were excluded. Korean national health system study, 1986-2001. GGT= $\gamma$ glutamyl transferase

attenuated the increased risk in the highest cholesterol group (fig $1)$.

The pattern was similar when we excluded events during the first five years of follow-up. Stratifying by presence or absence of hypertension showed that the inverse association with cholesterol was confined to people with hypertension (fig 2). In such participants, the hazard ratio for haemorrhagic stroke was 0.89 (0.84 to 0.94 ) for each $1 \mathrm{mmol} / \mathrm{l}$ increase in serum cholesterol. Participants without hypertension showed no evidence of increased risk of haemorrhagic stroke, but confidence intervals were wide.

GGT was positively associated with haemorrhagic stroke (2.02, 1.74 to 2.34 ; table 3$)$ and inversely associated with myocardial infarction, indicating a protective effect of alcohol. When stratified by GGT concentrations (low ( $\leq 45 \mathrm{U} / \mathrm{l}$ ), intermediate (46-80 U/l) and high ( $\geq 81 \mathrm{U} / \mathrm{l})$ ), the inverse association was confined to participants in the intermediate and high groups and was seen only in hypertensive participants (fig 3). When the analysis was stratified by self reported alcohol consumption ( $<30,30-209, \geq 210 \mathrm{~g}$ /week), the pattern was similar but the results were less clear than when stratified by GGT. The results were the same when we excluded $1 \%$ of the highest values for cholesterol and alcohol consumption.

\section{Discussion}

Our study included almost 4000 cases of haemorrhagic stroke, investigated a wide range of covariates including socioeconomic factors, and explored risk of haemorrhagic stroke at low concen- trations of cholesterol. We found an association between haemorrhagic stroke and low serum cholesterol only in participants with hypertension, suggesting that hypertension may modify the effects of low cholesterol.

The results of observational studies can be misleading because of cumulative effects of residual confounding. ${ }^{23}$ We believe our results are robust, given the specificity of the result, agreement with the randomised evidence, and adjustment for a wide range of covariates.

\section{The contribution of alcohol}

GGT reflects alcohol consumption and might be a better measure than self reporting. ${ }^{24}$ Raised GGT concentrations were caused mainly by hepatitis infection, alcohol consumption, and body mass index, so we excluded hepatitis carriers and adjusted for body mass index. Our finding that GGT was positively associated with haemorrhagic stroke and inversely associated with myocardial infarction agrees with earlier studies and validates

Table 3 Risk of stroke and myocardial infarction in Korean national health system study, 1986-2001. Values are fully adjusted hazard ratios (95\% confidence interval)

\begin{tabular}{lccc}
$\begin{array}{l}\gamma \text { glutamyl } \\
\text { transferase } \\
(\mathbf{U} / \mathbf{l})\end{array}$ & Ischaemic $(\mathbf{n}=\mathbf{2 0 6 7 )}$ & Stroke & $\begin{array}{c}\text { Myocardial infarction } \\
(\mathbf{n}=\mathbf{1 4 3 0})\end{array}$ \\
\cline { 2 - 3 } & 1.00 & 1.00 & 1.00 \\
\hline$\leq 45$ & $1.14(1.02$ to 1.27$)$ & $1.11(0.94$ to 1.32$)$ & $0.85(0.75$ to 0.98$)$ \\
\hline $46-80$ & $1.11(0.99$ to 1.25$)$ & $2.02(1.74$ to 2.34$)$ & $0.67 \quad(0.57$ to 0.79$)$ \\
\hline$\geq 81$ & &
\end{tabular}

Ischaemic stroke ICD I63, I67.8; haemorrhagic stroke ICD I61; myocardial infarction ICD |21-|24. 
the use of GGT as a marker of alcohol intake, especially for heavy drinkers. ${ }^{25}$ Previous studies have shown that people with low cholesterol concentrations are more likely to be heavy drinkers. ${ }^{26}$ Hypertension and other effects of alcohol could explain the increased risk of haemorrhagic stroke. ${ }^{27}$ In our study, increased risk of haemorrhagic stroke in people with low concentrations of blood cholesterol $(<4.14 \mathrm{mmol} / \mathrm{l})$ was restricted to those with high GGT values; this relation was less evident when alcohol consumption was measured by self report. The measures of blood pressure might not have been a true reflection of risk, as transient high blood pressure associated with binge drinking may have an important role in haemorrhagic stroke. ${ }^{25}$ At low concentrations of GGT, low serum cholesterol was not associated with a higher risk of haemorrhagic stroke. In effect, low blood cholesterol may act as a marker of the health damaging effects of alcohol, rather than be a cause of haemorrhagic stroke.

\section{Limitations}

All information was routinely collected. However, assessments of the quality of cholesterol assays are high. ${ }^{28}$

Errors may have been made in assigning the subtype of stroke. However, neuroimaging studies were conducted in $89 \%$ of hospital admissions for stroke between 1999 and 2000 in Korea. ${ }^{15}$ Since the early 1980 s, computed tomography has been incorporated into the routine diagnosis of stroke in Korea, and a high rate of neuroimaging can be expected in our study. ${ }^{29}$

The high ratios of stroke to myocardial infarction and haemorrhagic stroke to ischaemic stroke in our study are consistent with current data from Korea. Cause specific mortality was 73.8/ 100000 for stroke and 21.9/100 000 for myocardial infarction in 2001, and an independent study showed a similar composition of subtypes of stroke (143 cases (41\%) of haemorrhagic stroke and 205 cases (59\%) of ischaemic stroke among the validated 348 cases of stroke). ${ }^{30}{ }^{31}$

Our use of an occupational cohort might result in healthy worker effects (the general population includes healthy and sick people, whereas workers have at least a minimum level of health), although these should not influence associations between risk factors and outcomes. Heavy drinkers might have been excluded from the workforce, but this would have attenuated the observed associations.

\section{Implications}

Low blood cholesterol may not in itself increase risk of haemorrhagic stroke. Blood cholesterol values in the range likely to be achieved by treatment with statins are not associated with increased risk of haemorrhagic stroke. In countries with high rates of haemorrhagic stroke, preventive strategies that include lowering blood cholesterol should not be tempered because of concerns about a possible increased risk of haemorrhagic stroke.

Contributors: SE wrote the first draft of the paper and helped design, interpret, and revise the manuscript. JS and Y-MS had full access to the cohort data and are responsible for the integrity of data and accuracy of data analysis. JS helped design, interpret, and revise the manuscript and handle data. Y-MS helped design, analyse, and interpret the manuscript. RF helped interpret the results. DAL and GDS helped design the study and interpret the results. All authors commented on drafts and decided to submit for publication.JS and SE are guarantors.

Funding: Ministry of Health and Welfare Korea (01-PJ1-PG1-01CH100007) and MH63749 from the National Institutes of Mental Health. DAL is funded by a UK Department of Health career scientist award.

Competing interests: None declared.

Ethical approval: Internal review board of Samsung Medical Centre and Korea National Health Insurance Corporation.

1 Yusuf S, Reddy S, Ounpuu S, Anand S. Global burden of cardiovascular diseases. Part I. General considerations, the epidemiologic transition, risk factors and impact of urbanisation. Circulation 2001:104:2746-53.
2 Lawlor DA, Davey Smith G, Leon DA, Sterne J, Ebrahim S. Secular trends in mortality by stroke subtype in the 20th century: a retrospective analysis. Lancet 2002;360:181823.

Tuomilehto J, Bonita R, Stewart A, Nissinen A, Salonen JT. Hypertension, cigarette smoking, and the decline in stroke incidence in eastern Finland. Stroke 1991:22:7-11.

4 Vartiainen E, Sarti C, Tuomilehto J, Kuulasmaa K. Do changes in cardiovascular risk factors explain changes in mortality from stroke in Finland? BMJ 1995;310:901.

5 Shimamoto T, Komachi Y, Inada H, Doi M, Iso H, Sata S, et al. Trends for coronary heart disease and stroke and their risk factors in Japan. Circulation 1989;79:503-15.

6 Kubo M, Kiyohara Y, Kato I, Tanizaki Y, Arima H, Tanaka K, et al. Trends in the incidence, mortality and survival rate of cardiovascular disease in a Japanese community. The Hisayama study. Stroke 2003;34:2349-54

7 Ariesen MJ, Claus SP, Rinkel GJ, Algra A. Risk factors for intracerebral haemorrhage in the general population. A systematic review. Stroke 2003;34:2060-6.

8 Prospective Studies Collaboration. Cholesterol, diastolic blood pressure, and stroke: 13,000 strokes in 450,000 people in 45 prospective cohorts. Lancet 1995;346:1647-53.

Hart CL, Hole DJ, Davey Smith G. Risk factors and 20-year stroke mortality in men and women in the Renfrew/Paisley study in Scotland. Stroke 1999;30:1999-2007.

10 Wannamethee SG, Shaper AG, Ebrahim S. HDL-cholesterol, total cholesterol, and the risk of stroke in middle-aged British men. Stroke 2000;31:1882-8.

11 Neaton JD, Blackburn H, Jacobs D, Kuller L, Lee DJ, Sherwin R, et al. Serum cholesterol level and mortality findings for men screened in the multiple risk factor intervention trial. Multiple Risk Factor Intervention Trial Research Group. Arch Intern Med 1992;152:1490-500.

12 Yano K, Reed DM, MacLean CH. Serum cholesterol and hemorrhagic stroke in the Honolulu heart program. Stroke 1989;20:1460-5.

13 Hart CL, Hole DJ, Davey Smith G. The relation between cholesterol and haemorrhagic or ischaemic stroke in the Renfrew/Paisley study. J Epidemiol Community Health 2000;54:874-5

14 Asia Pacific Cohort Studies Collaboration. Zhang X, Patel A, Horibe H, Wu Z, Barzi F, Rodgers A, et al. Cholesterol, coronary heart disease and stroke in the Asia Pacific region. Int J Epidemiol 2003;32:563-72.

15 Suh I, Jee SH, Kim HC, Nam CM, Kim IS, Appel LJ. Low serum cholesterol and haemorrhagic stroke in men: Korea medical insurance corporation study. Lancet 2001:357:922-5.

16 Bots ML, Elwood PC, Nikitin Y, Salonen JT, Friere de Concalves A, Inzitari D, et al. Total and HDL cholesterol and risk of stroke. EUROSTROKE: a collaborative study among research centres in Europe.J Epidemiol Community Health 2002;56(suppl 1):119-24.

17 Iso H, Jacobs DR Jr, Wentworth D, Neaton JD, Cohen JD for the Multiple Risk Factor Intervention Trial Research Group. Serum cholesterol levels and six-year mortality from stroke in 350,977 men screened for the multiple risk factor intervention trial. $N$ Engl J Med 1989;320:904-10.

18 Corvol JC, Bouzamondo A, Sirol M, Hulot JS, Sanchez P, Lechat P. Differential effects of lipid-lowering therapies on stroke prevention: a meta-analysis of randomized trials. Arch Int Med 2003;163:669-76.

19 Cholesterol Treatment Trialists' (CTT) Collaborators. Baigent C, Keech A, Kearney PM, Blackwell L, Buck G, Pollicino C, et al. Efficacy and safety of cholesterol-lowering treatment: prospective meta-analysis of data from 90,056 participants in 14 randomised trials of statins. Lancet 2005:366:1267-78.

20 Song Y-M, Sung J, Kim JS. Which cholesterol level is related to the lowest mortality in a population with low mean cholesterol level: A 6.4-year follow-up study of 482,472 Korean men. Am J Epidemiol 2000;151:739-47.

21 Expert Panel on Detection, Evaluation, and Treatment of High Blood Cholesterol in Adults. Executive summary of the third report of the national cholesterol education program (NCEP) expert panel on detection, evaluation, and treatment of high blood cholesterol in adults (adult treatment panel III). JAMA 2001;285:2486-97.

22 Chobanian AV, Bakris GL, Black HR, Cushman WC, Green LA, Izzo JL Jr, et al, the National High Blood Pressure Education Program Coordinating Committee. The seventh report of the joint national committee on prevention, detection, and treatment of high blood pressure. JAMA 2003;289:2560-72.

23 Lawlor DA, Davey Smith G, Kundu D, Bruckdorfer KR, Ebrahim S. Those confounded vitamins: what can we learn from the differences between observational versus randomised trial evidence? Lancet 2004:363:1724-7.

\section{What is already known on this topic}

Low blood cholesterol may increase the risk of haemorrhagic stroke, raising concern that treatments that lower blood cholesterol may be harmful

Heavy alcohol consumption raises blood pressure, impairs liver function, and has anticoagulant effects and may underlie any association between low blood cholesterol and increased risk of haemorrhagic stroke

\section{What this study adds}

Increased risk of haemorrhagic stroke is confined to people with low concentrations of blood cholesterol and markers of high alcohol consumption

Lowering cholesterol is unlikely to increase the risk of haemorrhagic stroke, even in a population with a high incidence of haemorrhagic stroke 
24 Chen Z, Keech A, Collins R, Slavin B, Chen J, Campbell TC, et al. Prolonged infection with hepatitis $\mathrm{B}$ and association between low blood cholesterol concentration and liver cancer. BMJ 1993;306:890-4.

25 Reynolds K, Lewis LB, Nolen JD, Kinney GL, Sathya B, He J. Alcohol consumption and risk of stroke: a meta-analysis. JAMA 2003;289:579-88.

26 Iribarren C, Reed DM, Burchfiel CM, Dwyer JH. Serum total cholesterol and mortality: confounding factors and risk modification in Japanese-American men. JAMA 1995;273:1926-32.

27 Hart CL, Davey Smith G, Hole DJ, Hawthorne VM. Alcohol consumption and mortality from all causes, coronary heart disease and stroke: results from a prospective cohort of Scottish men with 21 years of follow up. BMJ 1999;318:1725-9.

28 Chung WS, Kim SH, Kim YS, Kim YK, Kim JQ, Yi KN, et al. Annual report on external quality assessment in clinical chemistry in Korea (1990). J Clin Pathol Qual Cont 1991;13:1-13.

29 Suh SI, Kim HJ, Park EC, Sohn M, Kim DK. The comparison of computerized tomography utilization between before and after coverage of medical insurance. Korean J Hos Management 1997:3:121-33.

30 Korea National Statistical Office. Annual report on the cause of death statistics (based on vital registration). Daejeon, Republic of Korea: Korea National Statistical Office, 2002

31 Park JK, Kim KS, Kim CB, Lee TY, Lee KS, Lee DH, et al. The accuracy of ICD codes for cerebrovascular diseases in medical insurance claims. Korean J Prev Med 2000:33:76-82

(Accepted 14 April 2006) doi $10.1136 / \mathrm{bmj} .38855 .610324 .80$

Department of Social Medicine, University of Bristol, Bristol BS8 2PR

Shah Ebrahim epidemiologist

Debbie A Lawlor epidemiologist

George Davey Smith epidemiologist

Department of Preventive Medicine, Kangwon National University College of Medicine, Kangwon-Do, Korea

Joohon Sung epidemiologist

Department of Family Medicine, Samsung Medical Centre, SungKyunKwan University School of Medicine, Seoul, Korea

Yun-Mi Song epidemiologist

Department of Family and Community Medicine, University of Texas Health Science Center at San Antonio, San Antonio, TX, 78229, USA

Robert L Ferrer epidemiologist

Correspondence to: J Sung, Department of Preventive Medicine, Kangwon National University College of Medicine, Hyoja-2-Dong, Chunchon, Kangwon-Do, Korea sungjohn@kangwon.ac.kr or sungjohn@empal.com 